\title{
A New Method to Calibrate Induction Magnetometers
}

\author{
L.F. Zambresky, T. WATANABe, R.D. Russell, T. OGuti, ${ }^{*}$ \\ and K. HAYASHI \\ Department of Geophysics and Astronomy, University of \\ British Columbia, Vancouver, B.C., Canada
}

(Received October 16, 1978; Revised March 5, 1979)

\begin{abstract}
A new method for calibrating induction magnetometers has been investigated theoretically and experimentally. The method is to construct a Wheatstone bridge using the head sensor coil of a magnetometer as one arm and to drive it externally with an electrical oscillator. It has been proved analytically that the voltage output from the load of the bridge is proportional, under appropriate conditions, to the e.m.f. induced in the head sensor coil by an external magnetic field which changes with time in the same manner as the driving voltage of the oscillator. The proof presented here is based on linear circuit theory; a later publication will consider nonlinearities.

The amplitude response of the bridge plus amplifier system has been determined experimentally and compared with the amplitude response of the system calculated theoretically, assuming an equivalent circuit of the head sensor. Agreement between theory and experiment is excellent. The calibration method is technically simple and can easily be employed for field work.
\end{abstract}

\section{Introduction}

An induction type magnetometer, among many different types of magnetometers, is the one used most often for observation of magnetic pulsation. A usual induction magnetometer consists of two parts connected in tandem: a coil used as a head sensor and an amplifier-recorder system. Two types of head sensor coils are commonly used: an air-core loop antenna and a solenoid having a high- $\mu$ metal core. The calibration method described in this paper is to construct a Wheatstone bridge using the head sensor as one of the bridge's arms (Fig. 1). The bridge is then driven externally by an electrical oscillator. As will be seen later, this bridge arrangement correctly simulates magnetic excitation of the induction magnetometer in the operation mode for observation.

The original idea of the bridge method stemmed from previously successful undertakings to calibrate an electromechanical seismometer using a Maxwell bridge (e.g., Willmore, 1959; Kollar and Russell, 1966). A general theory

\footnotetext{
* On leave from the Geophysics Research Laboratory, University of Tokyo, Tokyo, Japan.
} 
of the bridge method for induction magnetometers has been developed by Russell and Watanabe (1978), assuming non-linearities in self-inductance and resistance of the sensor. The non-linearities have to be taken into consideration when using high- $\mu$ metal core coils as a head sensor. In practice, however, the non-linear effect do not seem to be significant (UEDA and WATANABE, 1978), and in this paper we assume a linear sensor. A theory of the bridge method under the linearity hypothesis is developed in Section 2. The results of a laboratory experiment is given in Section 3. The agreement between theory and experiment is excellent. The method is simple technically and offers a practical means for calibration both in the laboratory and in the field.

\section{Principles of the Bridge Method}

The circuit diagram for the bridge method is shown in Fig. 1. The equivalent circuit of the sensor coil is depicted as a capacitor connected in parallel with the series combination of a resistor, an e.m.f. source and an inductor (Fig. 2). That this equivalent circuit accurately describes the action of the sensor has been established experimentally (CAMPBELL, 1969; UEDA and WATANABE, 1975a; Zambresky and WATANABe, 1978). The e.m.f. $N \dot{\phi}$ in the sensor coil arises from natural magnetic field variations. Here $N$ is the total number of turns and $\dot{\phi}$ the average flux enclosed. When a calibration is being performed, a signal generator, $v$, is used to drive the bridge. The bridge is balanced for D.C., whether the detection system of Fig. 1 is in the operation mode $(v=0)$ or in the calibration mode $(\dot{\phi}=0)$ :

$$
R R_{C}=R_{B} R_{R}
$$

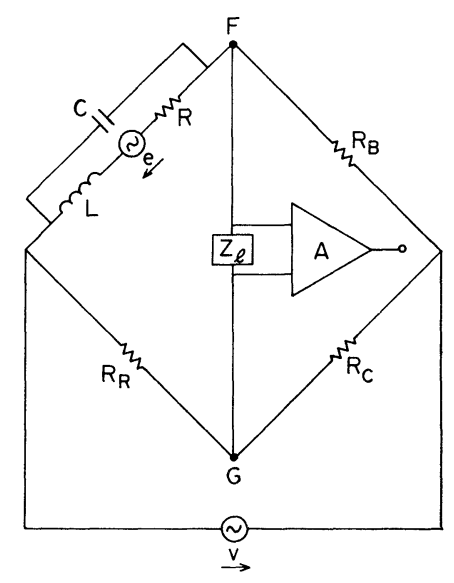

Fig. 1. Circuit of the bridge method. 
For an A.C. source, $v$, the balance condition will be violated, since the impedance of the arm of the sensor is not $R$, a constant, but dependent also on $L, C$ and $\omega$, the angular frequency of the A.C. source. The load current will no longer be zero, but should depend on $R, L$ and $C$, the electric constants of the sensor. These constants determine the frequency response of the sensor. Therefore, it is expected that the load current carries information on the sensor's frequency response. In what follows, it will be shown analytically that under appropriate conditions the load current truly reflects the frequency response of the sensor.

First consider the case when frequencies are low enough so that the distributed capacitance of the sensor is negligible. Then apply simultaneously an external field to provide a flux $\dot{\phi}$ in the sensor core and a potential $v$ from the oscillator. Suppose that these two stimuli combine to give a null current through the load. Then we know that $-v$ simulates a field giving a flux equal to $\dot{\phi}$.

Algebraically, in the frequency domain where we use $s$ as an abbreviation for $j \omega$, the voltages at the terminals of the load, $F$ and $G$, are given as follows,

$$
\begin{aligned}
& v_{F}=\frac{R_{B}(v+N \dot{\phi})}{R_{B}+R+s L} \\
& v_{G}=\frac{R_{C} v}{R_{C}+R_{R}}
\end{aligned}
$$

where the reference for $v_{F}$ and $v_{G}$ is at the junction of $R_{B}$ and $R_{C}$. Of course, $\dot{\phi}$ and $v$ are assumed to be sinusoidal in time change. Since no load current flows

and therefore

$$
v_{F}=v_{G}
$$

$$
N \dot{\phi}\left(\frac{R_{B}}{R_{B}+R+s L}\right)=v\left(\frac{R_{C}}{R_{C}+R_{R}}-\frac{R_{B}}{R_{B}+R+s L}\right) .
$$

Suppose further that the condition is met that the bridge is balanced at D.C. (Eq. (1)). Then, the previous equation reduces to

$$
N \dot{\phi}=\frac{s L v}{R_{B}+R}=\frac{L \dot{v}}{R_{B}+R} .
$$

Thus there is an exact correspondence between $\dot{v}$ and $\dot{\phi}$. Since $\phi$ is related to $H$, the strength of the external magnetic field, by the magnetic properties and geometry of the sensor (presumed constant), it is clear that a voltage $v$ simulates $H$. The absence of $s$ in Eq. (5) means that it is valid for all frequencies and, indeed, for non-sinusoidal stimuli. 

Then,

At higher frequencies the distributed capacitance $C$ cannot be ignored.

and

$$
v_{F}=v+\frac{N \dot{\phi} R_{B}-(s L+R) v}{R_{B}\left(s^{2} L C+s C R+1\right)+s L+R}
$$

$$
v_{G}=\frac{R_{C} v}{R_{C}+R}
$$

But, as before, $v_{F}=v_{G}$, and assuming the D.C. balance of the bridge (Eq. (1)), we have

$$
N \dot{\phi}=\frac{s L v}{R_{B}+R}\left(1-\frac{C R^{2}}{L}-s C R\right) .
$$

If the $Q$ of the sensor is high (as it is in the particular sensor studied here), then $L \gg C R^{2}$, because the $Q$ is defined by

$$
Q=\frac{1}{R} \sqrt{\frac{L}{C}}
$$

and Eq. (8) is simplified to

$$
N \dot{\phi}=\frac{s L v}{R_{B}+R}(1-s C R)
$$

i.e.,

$$
N \dot{\phi}=\frac{L \dot{v}}{R_{B}+R}(1-s C R) .
$$

In this case, where $C$ cannot be ignored, the relationship between $\phi$ and $v$ (and hence the relationship between $H$ and $v$ ) is specifically frequency dependent. Therefore, Eq. (11) can only be easily used for sisusoidal signals.

Even if $Q$ is not high enough, we see from Eq. (8) that the ratio of $\phi$ to $v$ is still a linear function of frequency, as far as $Q \neq 1$. The constants $L, C$ and $R$, and hence $Q$ are easily measurable quantities (UEDA and WATANABE, 1975a). Air core loop sensors have generally lower $Q$ values than high- $\mu$ metal core sensors. An equation of the form of (11) can still be achieved exactly even in the case where $Q$ is not high if one inserts a compensating inductance $L^{\prime}=R_{C} R C$ into the arm of resistance $R_{C}$ (Russell and WATANABE, 1978).

If the bridge components are chosen so that $R_{B} \gg R$, then possible nonlinearities of the sensor resistance $R$ are unimportant. Furthermore, for practical sensor applications, the term $s C R$ is small with respect to 1 .

As noted above, the average magnetic flux crossing a coil winding is $\phi$ and the coil is wound with $N$ turns of wire; the total magetic flux crossing the entire windings of the sensor coil is given by $N \phi$. This is related to the absolute sensitivity of the sensor coil, $S_{a}$ as follows (UeDA and Watanabe, 1975b), 


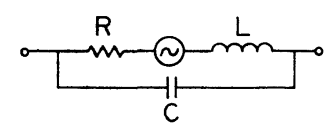

Fig. 2

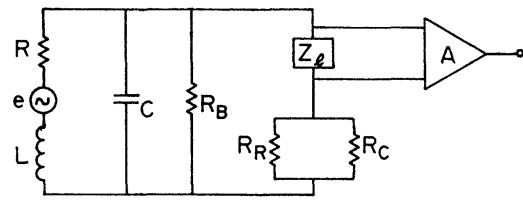

Fig. 3

Fig. 2. Equivalent circuit of a head sensor coil.

Fig. 3. Equivalent circuit of the bridge system in the operation mode $(v=0)$.

$$
N \phi=S_{a} H
$$

where $H$ is the component of the varying natural magnetic field parallel to the axis of the sensor coil. The e.m.f., $e$, induced in the sensor coil is relate to the total magnetic flux as follows,

$$
N \dot{\phi}=S_{a} \dot{H}
$$

Therefore, Eq. (5) can be rewritten as

or $\left(R_{B} \gg R\right)$ as

$$
v=\frac{\left(R+R_{B}\right)}{L} N \phi
$$

$$
v \cong \frac{R_{B}}{L} S_{a} H
$$

In the last two equations, $v$ is the driving voltage simulating the external field, $-v$ including the sign correctly. Either of these two equations shows that the total magnetic flux through the sensor is simulated by the bridge arrangement.

If the system of Fig. 1 is in the operation mode, the signal generator is replaced with a short circuit. Then, the circuit of Fig. 1 is reduced to the one shown in Fig. 3. Considering Fig. 3, two alternatives for practical operation present themselves. One is to provide each sensor with its own bridge components. In this way the circuit used including the attenuation caused by the bridge components is exactly the circuit to which the calibration applies. Alternatively the bridge components can be removed after calibration and the calibration adjusted to compensate for the removal of the bridge components. This adjustment will be small if

and

$$
R_{B} \gg\left|Z_{l}+R_{R} / / R_{C}\right|
$$

$$
\left|Z_{l}\right| \gg R_{R} / / R_{C}
$$

where $R_{R} / / R_{C}$ is an abbreviation for the parallel resistance of $R_{R}$ and $R_{C}$.

Reduction of the bridge circuit of Fig. 1 to the equivalent circuit shown in Fig. 3 is based on an assumption that the output impedance of the oscillator 


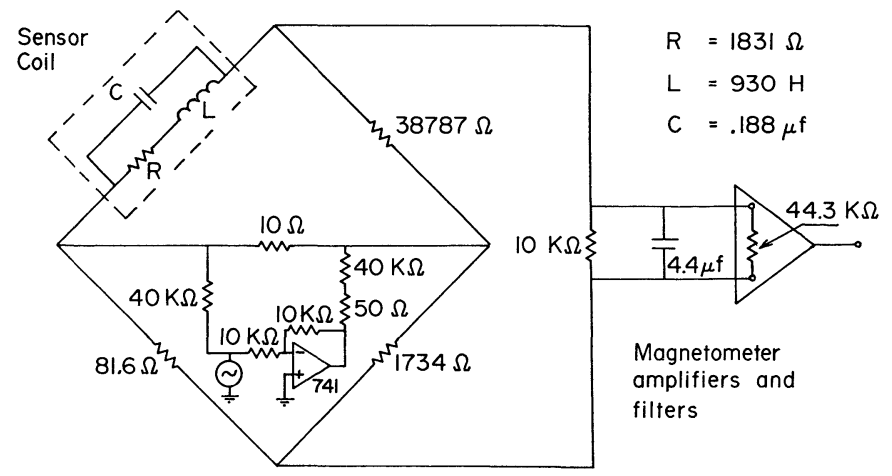

Fig. 4. Experimental setup of the bridge method.

$v$ is zero. In actuality, it is not zero but $10 \Omega$ (see Fig. 4). However, it has been confirmed through numerical analysis that the effects of this non-zero output impedance is negligible.

\section{Experimental Results}

The results of a laboratory experiment to determine the amplitude versus frequency response by the bridge method are presented in this section. The actual circuit arrangement for the experiment is shown in Fig. 4. The bridge resistors were so determined as to satisfy Eq. (1) and the condition $R_{B} \gg R_{R}$. The resistance of the $R_{C}$ arm was made approximately equal to $R$, the resistace of the sensor. The resistance of the $R_{R}$ arm was taken as follows: $R_{R}=R / 20$, and the bridge was adjusted to zero d.c. output by the variable resistor $R_{B}$. Also, it must be noted that an inverting amplifier was used as part of the circuit to feed signals to the bridge. This arrangement was made to meet the condition that the first stage of the amplifier-a chopper stabilized d.c. amplifier-requires a balanced in put signal. A sinusoidal signal $v$ was fed to the bridge by the oscillator. The amplitude of $v$ was held constant while its frequency was changed. Then as seen from Eq. (15), the driving voltage, $v$, then simulates sinusoidal geomagnetic field variations which have a constant amplitude but different frequencies. The value of $Q^{2}$ of the sensor is 1,500 approximately and $\omega C R$ is not more than 0.001 over the entire frequency range of interest.

The experimental results are shown in Fig. 5. The solid circles in Fig. 5a represent voltage readings at the output terminal of the amplifier for different frequencies. The d.c. gain of the whole amplifying system is 200,000 . The data points were normalized to the output at a frequency of $0.1 \mathrm{~Hz}$. The actual output at a frequency of $1 \mathrm{~Hz}$ was approximately 13 volts peak-to-peak. The 


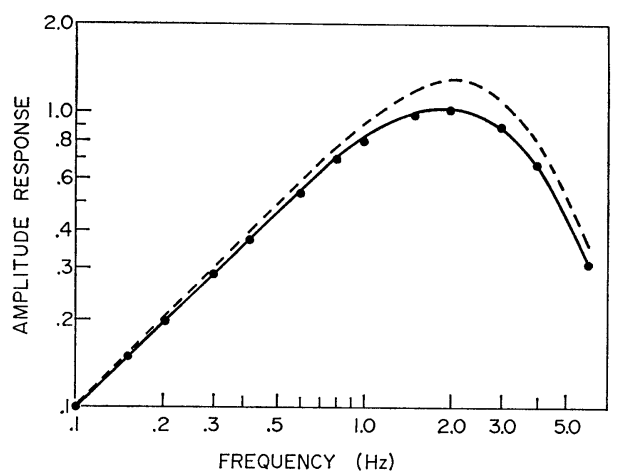

(a)

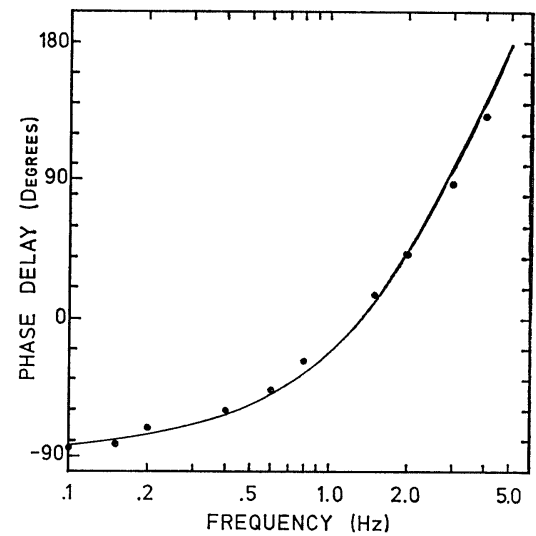

(b)

Fig. 5. Amplitude response (a) and phase response (b) of the bridge plus amplifier/ recorder system. Solid circles represent the experimental results and the solid curve a theoretical prediction. The dotted curve in (a) shows the theoretical amplitude response of the system when the sensor is directly connected to the amplifier without forming a bridge.

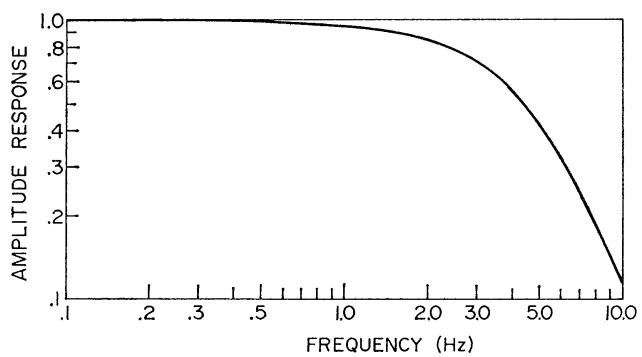

(a)

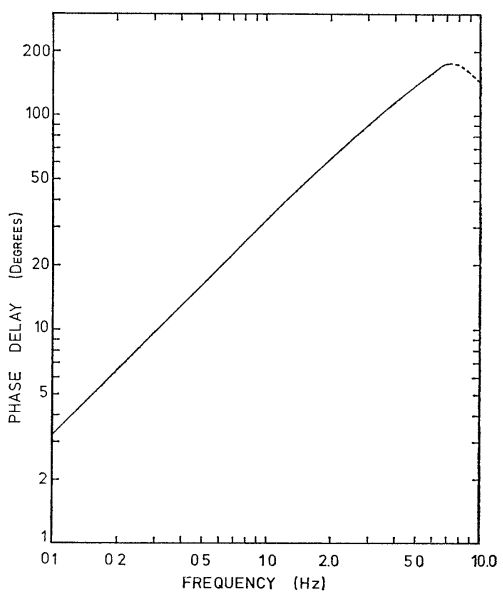

(b)

Fig. 6. Amplitude response (a) and phase response (b) of the amplifier only. In (b), the dotted portion of the curve represents phase advance rather than phase delay.

smooth curve shows the amplitude response calculated theoretically; it is a plot of the theoretical transfer function of the whole bridge-amplifier system. Agreement between theory and experiment is excellent. This corresponds to an external sinusoidal magnetic field variation of a peak-to-peak amplitude of 
Table 1. Characteristics of the head sensor.

\begin{tabular}{|c|c|c|c|}
\hline Resistance $(R)$ & $1,831 \Omega$ & Turn number $(N)$ & 50,000 \\
\hline Self-inductance $(L)$ & $930 \mathrm{H}$ & Coil length & $45.7 \mathrm{~cm}$ \\
\hline Distributed-capacitance $(C)$ & $.188 \mu \mathrm{F}$ & Coil diameter, inner & $3.2 \mathrm{~cm}$ \\
\hline \multirow[t]{2}{*}{ Absolute sensitivity $(R)^{*}$} & $.0088(\mu \mathrm{V} / \mathrm{pT} / \mathrm{s})$ & outer & $7.3 \mathrm{~cm}$ \\
\hline & .055 Caner & Length of core & $91.4 \mathrm{~cm}$ \\
\hline
\end{tabular}

* Refer to HAYASHI et al. (1978) concerning the absolute calibration of the sensor. Refer to UEDA and WATANABE (1975b) about Caner. The sensitity of a coil is said to be 1 Caner if an e.f.m. of 1 microvolt in amplitude is induced by an sinusoidal external magnetic field, parallel to the coil's axis, of 1 milligamma ( 1 picotesla) in amplitude and of $1 \mathrm{~Hz}$ in frequency.

approximately $1.9 \gamma$. The theoretical transfer function was the product of the transfer function of the bridge and that of the amplifier system. The transfer function of the bridge was calculated from the equivalent circuit shown in Fig. 3 in which the e.m.f., $e$, was assumed to be proportional to frequency. The transfer function of the amplifier only is shown in Fig. 6. The recorder used for this experiment has a flat response over the entire range of frequency of interest to us.

The dotted curve in Fig. 5a shows the amplitude response of the system when the sensor is directly connected to the amplifier across the load impedance. It consists of a composite resistance $10 \mathrm{k} \Omega / / 44.3 \mathrm{k} \Omega$ and a parallel capacitor of $4.4 \mu \mathrm{F}$ (see Fig. 4). The data points at lower frequencies agree with the dotted curve but not in a frequency range centered at $2 \mathrm{~Hz}$. The discrepancy is not surprising, however. It indicates the effects of the bridge arms, $R_{R}, R_{C}$ and $R_{B}$. The dotted curve corresponds to the case when $R_{B} \rightarrow \infty$ and $R_{C} \rightarrow 0$ in Fig. 3 (see also the inequalities (16) and (17)).

If the sensor is terminated with the load impedance $Z_{l}$ without forming a bridge (the case of the dotted curve in Fig. 5a), the sensor plus $Z_{l}$ systems behaves approximately as if it is a Butterworth filter of the second degree alternating the e.m.f. It has been demonstrated by theory and by experiment that terminating a sensor with a set of properly chosen resistance and capacitor makes the sensor's response a Butterworth type (ZAMBresKy and WATANABE, 1978). With choice made for this experiment, the Butterworth corner frequency becomes approximately $2 \mathrm{~Hz}$. This is why the response in amplitude becomes approximately maximum at this frequency.

The theoretical phase of the sensor-bridge plus the amplifier system (Fig. 4) is shown with a solid curve in Fig. 5b. The solid circles represent the experimentally obtained phase delays of the output voltage compared to the driving voltage $v$ which simulates the external magnetic field variation. The phase differences were obtained comparing the waveforms of the driving and output 
signals recorded on a 2-channel pen-writing recorder. Each individual experimental value agrees well with the theoretical prediction. The largest deviation is not more than 10 degrees. The rms error is 5 degrees approximately.

\section{Conclusions}

The bridge method for calibrating a geomagnetic sensor has been demonstrated to be sound in theory and to be simple in practice. An alternative method for calibrating an induction magnetometer is to create a time-changing magnetic field which is to be applied externally to the head sensor. The external field has to be uniform in space, considerably longer in every dimension compared to the length of the coil and/or the core of the head sensor. Theoretically, the field has to be uniform in 'infinite' space. To create an extensive uniform magnetic field is not too easy in practice. The bridge method is much easier in this respect. The method by an external magnetic field has a merit, however; it can calibrate the absolute sensitivity of the head sensor. Absolute calibration by the bridge method has been proved to be possible with regard to a spheroidshaped high- $\mu$ metal core coil (Russell and Watanabe, 1978). An alternative method for absolute calibration is to adopt a well-calibrated magnetometer as a standard and to run it simultaneously with an induction magnetometer with an unknown sensitivity. An air-core loop antenna can serve as such a standard. The absolute sensitivity of an air-core loop antenna can be determined by calculation, knowing the geometry of the loop and the total turn number of the coil's winding. Absolute sensitivity of a high- $\mu$ metal core coil, identical to the one used for this experiment, has been carried out successfully by this technique (HAYASHI et al., 1978).

A disadvantage of the bridge method is that the bridge's arms other than the sensor affect the response of the sensor itself. The amplitude response of a system when the sensor is constructed into the bridge, must be different from that of a system when the sensor is connected directly to the amplifier (see Fig. 5a). The discrepancy can be made very small assigning an extremely small resistance to the arm $R_{R}$ of the bridge. This implies that the resistance of the arm $R_{B}$ should be extremely large. It is difficult to secure the bridge's balancing condition, viz. Eq. (1), with such extreme resistance values. It does not seem to be practical to reduce the discrepancy in this way. It may be preferable to leave the bridge as a permanent part of the sensor system. Alternatively, we can connect the sensor directly to the amplifier without constructing a bridge and calculate the system response theoretically. Even in this case, it is useful to carry out calibration by the bridge method once and to confirm that the theoretically predicted response curve agrees with an experimental result. Such 
a test renders a great deal of confidence in the theoretical procedure to determine the system response.

The theoretical response is obtained by calculating the transfer function of the system, the sensor with or without a bridge plus the amplifier. The transfer function is a complex function. It yields not only the amplitude response but also the phase response.

The authors sincerely appreciate technical information provided by Dr. L.K. Law, the Victoria Geophysical Observatory, Victoria, B.C. and by Dr. J.E. Lokken and Mr. J.A. Shand of the Defense Research Establishment Pacific, Esquimalt, B.C. The authors also acknowledge with thanks assistance of the technical staff of the Dept. of Geophysics and Astronomy, University of British Columbia (abbrev. with U.B.C.). This investigation was supported by the National Research Council of Canada under Grants A-3564 and E-2923. Sojourn of the two authors (T.O. and K.H.) at U.B.C. was also supported by the National Research Council of Canada.

\section{REFERENCES}

CAMPBeLL, W.H., Induction loop antennas for geomagnetic field variation measurements, Technical report, ERL 123-ESL6, Environment Sciences Services Administration, Boulder, Colo., U.S.A., 1969.

Hayashi, K., T. Oguti, T. Watanabe, and L.F. Zambresky, Absolute sensitivity of a high- $\mu$ metal core solenoid as a magnetic sensor, J. Geomag. Geoelectr., 30, 619-630, 1978.

Kollar, F. and R.D. Russell, Seismometer analysis using an electric current analog, Bull. Seism. Soc. Am., 56, 1193-1205, 1966.

Russell, R.D. and T. Watanabe, A proposal for a bridge method for the calibration of geomagnetic sensor, J. Geomag. Geoelectr., 32, 1980 (in press).

Ueda, H. and T. WATANABE, Comments on the anti-resonance method to measure the circuit constants of a coil used as a sensor of an induction magnetometer-Technical notes, Sci. Rept. Tohoku Univ., Ser. 5, Geophys., 22, 129-135, 1975a.

Ueda, H. and T. Watanabe, Several problems about sensitivity and frequency response of an induction magnetometer, Sci. Rept. Tohoku Univ., Ser. 5, Geophys., 22, 107-127, 1975b.

Ueda, H. and T. WATANABE, Linearity of a ferromagnetic core solenoid as a magnetic sensor, J. Geomag. Geoelectr., 32, 1980 (in press).

Willmore, P.L., The application of the Maxwell impedance bridge to the calibration of electromagnetic seismograph, Bull. Seism. Soc., Am., 49, 99-114, 1959.

ZAMBRESKY, L.F. and T. WATANABE, Equivalent circuit of a magneric sensor coil and a simple filter for rejection of $60 \mathrm{~Hz}$ man-made noise, J. Geomag. Geoelectr., 32, 1980 (in press). 